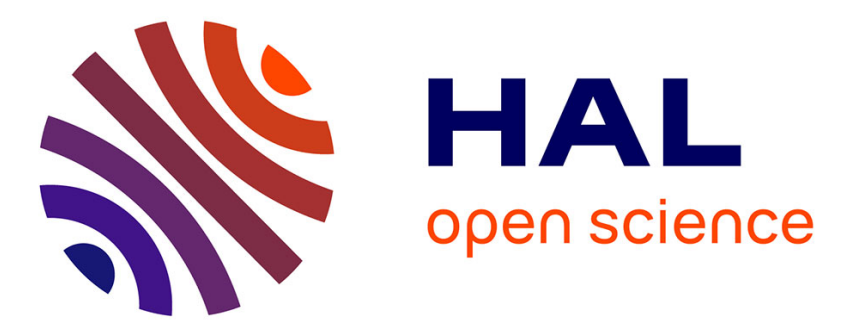

\title{
La concurrence des souffrances. Genèse et usages électifs de la catégorie des orphelins et enfants vulnérables au temps du sida
}

Fabienne Hejoaka

\section{- To cite this version:}

Fabienne Hejoaka. La concurrence des souffrances. Genèse et usages électifs de la catégorie des orphelins et enfants vulnérables au temps du sida. Autrepart - Revue de sciences sociales au Sud, 2014, 72 (4), pp.59. 10.3917/autr.072.0059 . hal-02868686

\section{HAL Id: hal-02868686 \\ https://hal-amu.archives-ouvertes.fr/hal-02868686}

Submitted on 15 Jun 2020

HAL is a multi-disciplinary open access archive for the deposit and dissemination of scientific research documents, whether they are published or not. The documents may come from teaching and research institutions in France or abroad, or from public or private research centers.
L'archive ouverte pluridisciplinaire HAL, est destinée au dépôt et à la diffusion de documents scientifiques de niveau recherche, publiés ou non, émanant des établissements d'enseignement et de recherche français ou étrangers, des laboratoires publics ou privés. 


\title{
La concurrence des souffrances. Genèse et usages électifs de la catégorie des orphelins et enfants vulnérables au temps du sida
}

\author{
Fabienne Hejoaka*
}

Du 25 au 27 juin 2001 s'est tenue à New York une session extraordinaire de l'assemblée générale des Nations unies consacrée pour la première fois, dans l'histoire de l'organisation internationale, à une maladie : l'infection à VIH. La Déclaration d'engagement produite à l'issue de cet événement comprenait, à côté des articles consacrés au genre, à l'accès aux traitements ou à la stigmatisation, trois articles plus particulièrement dédiés aux « orphelins et enfants rendus vulnérables par le VIH/sida ». L'infection à VIH ayant longtemps été pensée comme une épidémie d'adultes [Hejoaka, 2012], ces articles marquaient l'inscription de la question des enfants sur l'agenda des politiques internationales de lutte contre le sida. Ils entérinaient également l'institutionnalisation de cette nouvelle catégorie de l'enfance globalisée que sont les « orphelins et enfants vulnérables » $(\mathrm{OEV}){ }^{1}$.

La catégorie des OEV offre un exemple paradigmatique des dynamiques sociohistoriques et des usages politiques et sociaux qui sous-tendent la fabrication institutionnelle de catégories contemporaines de l'enfance dans le cadre de l'aide internationale $^{2}$. Cette catégorie fait partie d'un ensemble de catégories politicoadministratives produites $a d$ hoc à partir de la fin des années 1980, en réponse à l'impact de l'épidémie sur les enfants en Afrique subsaharienne ${ }^{3}$. Les OEV ne constituent pas une catégorie transhistorique instituée spontanément au début de l'épidémie. À la fin des années 1980, les politiques et les programmes d'aide ont essentiellement ciblé les orphelins du sida. La catégorie OEV n'a été instituée

\footnotetext{
* Anthropologue, post-doctorante à l'IRD (UMI 233 TransVIHMI) et chercheuse associée au Centre de recherche clinique et de formation de Fann (Dakar, Sénégal)

1. En 2012, selon l'Onusida [2013], en Afrique subsaharienne, 15,1 millions d'enfants âgés de moins de 18 ans étaient orphelins des suites du VIH/Sida, tandis que 2,9 millions d'enfants âgés de 0 à 14 ans étaient infectés par le VIH [Onusida, 2013].

2. Intégrant les évolutions et reconfigurations des mondes du développement et de l'aide urgence intervenues ces trois dernières décennies, cet article s'inscrit dans la définition de l'aide internationale proposée par Atlani-Duault et Dozon [2011], qui « fait simultanément référence aux faits de développement et de l'action humanitaire. »

3. Les autres catégories sont les « orphelins du sida », les « enfants affectés », les « enfants infectés », les «enfants exposés» ou les «adolescents vulnérables au VIH/sida ».
} 
qu'une décennie plus tard, en réponse aux critiques adressées à la catégorie des « orphelins du sida ».

Analysant la genèse et les effets à la fois paradoxaux et électifs de la fabrication de catégories institutionnelles à partir du cas des OEV, cet article offre une série de réflexions sur le traitement des enfants dans les programmes d'aide internationale ${ }^{4}$. Penser cet objet invite notamment à décrire comment les catégories produites par les politiques internationales et les mobilisations sociales vivent, se modifient voire s'éteignent selon des processus sociaux de fabrication et d'évolution, contingents ayant leurs dynamiques propres. Par ailleurs, les catégories institutionnelles, en tant que cadres normatifs à travers lesquels l'action publique locale et internationale est mise en œuvre, informent les pratiques interventionnelles et structurent la manière dont elles sont perçues. Comme l'analyse Wells dans son ouvrage Childhood in global perspectives [2009], au-delà de la culture et du contexte social local qui façonnent les enfants, leur vie est aujourd'hui construite et conditionnée par les politiques internationales de l'enfance. Ainsi, à côté d'une approche constructionniste permettant de décrire le processus de production d'un objet par l'action publique, en l'occurrence une catégorie institutionnelle, il est nécessaire de s'intéresser aux usages et aux effets politico-sociaux de ces catégorisations, en décrivant empiriquement «à niveau d'enfant», ce que les catégories institutionnelles font aux enfants. Dans le cas des OEV, l'analyse microsociale du traitement des enfants dans les programmes de lutte contre le sida révèle une tension que je qualifie de «concurrence des souffrances» entre les enfants bénéficiaires des programmes et ceux qui en sont exclus.

Cet article s'appuie sur des travaux ethnographiques menés entre 2005 et 2008 au Burkina Faso sur le traitement politique, moral et médical des enfants dans la réponse apportée à l'épidémie du sida ${ }^{5}$. Il s'inscrit à l'intersection de la nouvelle sociologie de l'enfance [James, Prout, 1990] et de l'anthropologie critique de l'aide internationale, champ de recherche dynamique en expansion où s'entrecroisent les problématiques du développement et de l'aide humanitaire dans un contexte de mondialisation [Atlani-Duault, Vidal, 2009 ; Pandolfi, Corbet, 2011]. Toutefois, alors que les enfants et les adolescents sont des composantes démographiques majeures des sociétés subsahariennes et représentent une cible privilégiée de l'aide humanitaire, l'anthropologie francophone s'est peu intéressée à la question des enfants. Ceci s'explique notamment par le fait que l'anthropologie de l'enfance constitue un sous-champ méconnu et faiblement institutionnalisé d'une

4. Je remercie vivement pour leur lecture d'une version précédente de cet article et leurs suggestions, Maryvonne Charmillot (Équipe ACRA, université de Genève), Philippe Msellati (IRD) et Caroline Desclaux-Sall (IRD). L'article a également grandement bénéficié des critiques pertinentes et constructives des évaluateurs anonymes.

5. Cet article est issu d'une recherche doctorale en anthropologie portant sur les politiques de lutte contre le sida et l'expérience de la maladie vécue par les enfants vivant avec le VIH au Burkina Faso [Hejoaka, 2012]. Réalisée au Centre d'études africaines (CEAf) à l'EHESS Paris, en association avec l'Institut de recherche pour le développement (UMI 233, TransVIHMI), cette recherche a bénéficié du soutien financier de l'Agence nationale de recherche sur le VIH/sida et les hépatites (bourse prédoctorale de 2005 à 2008), et de Sidaction (bourse prédoctorale de 2008 à 2009). 
discipline essentiellement focalisée sur les adultes. Les enfants ont longtemps incarné de «petits sujets » dans le champ anthropologique, comme l'ont décrit Lallemand et Le Moal [1981]. Toutefois, s'ils occupent une place marginale au sein de la discipline, les enfants et l'enfance font l'objet d'une vaste littérature anthropologique (par exemple Bonnet [2012], Lancy [2008]). Il existe ainsi une longue tradition d'études ethnographiques des enfants et des adolescents dans le contexte des pays des Suds, notamment subsahariens, qui offrent des données empiriques et théoriques fondamentales pour penser et comprendre les enfants à l'intersection du local et du global. Mais à ce jour, ces travaux francophones ont peu traité de la place des enfants en lien avec l'aide internationale.

L'article est structuré en trois parties. La première propose une sociohistoire de la genèse de la catégorie des OEV dans le contexte de l'épidémie de sida. Elle décrypte l'émergence et l'institutionnalisation de classifications, dispositifs, pratiques et discours des différents acteurs internationaux et locaux en réponse aux conséquences de l'épidémie sur les enfants. À partir de la description microsociale d'une famille affectée par le VIH, la deuxième partie propose une vignette ethnographique décrivant empiriquement la concurrence des souffrances et les usages paradoxaux inhérents à l'opérationnalisation de la catégorie des $\mathrm{OEV}$. La troisième partie présente trois leçons qui peuvent être tirées de l'analyse critique et empirique de la fabrique institutionnelle des $\mathrm{OEV}$. Elle met en exergue la nécessité d'une approche générationnelle, l'existence du «triage institutionnel » opéré par les catégorisations et enfin, la nécessité de dépasser une approche monolithique des enfants dans les politiques et programmes d'aide internationale.

\section{La fabrique de la catégorie des OEV : genèse, tensions et institutionnalisation}

\section{Les « orphelins du sida » : de la crise sociale à la fabrique d'une catégorie institutionnelle}

À partir de la fin des années 1980, l'épidémie de sida hyperendémique ${ }^{6}$ qui a touché les pays d'Afrique Centrale et de l'Est s'est traduite par une augmentation exponentielle du nombre d'enfants orphelins ${ }^{7}$. L'infection à VIH touchant majoritairement des populations en âge de procréer, des pays comme l'Ouganda et la Tanzanie ont été confrontés à une « crise des orphelins du sida », comme cela a été qualifié dans la presse et par les institutions internationales [Foster, Germann, 2002]. Dans un contexte de transformation et d'épuisement des réseaux familiaux d'entraide, de solidarité et de confiage au sein de la famille élargie [Nyambedha,

6. Selon l'Onusida, le terme d'épidémie hyperendémique est employé pour qualifier les épidémies généralisées d'Afrique australe qui présentent une prévalence élevée et durable atteignant habituellement $15 \%$ ou davantage chez les femmes enceintes bénéficiant d'un suivi prénatal [Unaids, 2011].

7. L'efficacité des traitements antirétroviraux hautement actifs permettant de contrôler l'infection à VIH a été mise en évidence en 1996. Si les médicaments ont été rapidement disponibles dans les pays du Nord, l'accès aux antirétroviraux n'a été mis en œuvre en Afrique subsaharienne à une échelle nationale qu'à partir des années 2000. 
Wandibba, Aagaard-Hansen, 2003], nombre d'enfants orphelins se retrouvèrent privés d'éducation, d'affection et de soutien parental, d'une alimentation adéquate, voire d'hébergement. Les associations internationales et locales présentes sur le terrain furent les premiers acteurs institutionnels à élaborer des programmes pour répondre aux besoins vitaux de ces orphelins. Certaines jouèrent également un rôle pionnier dans les mobilisations collectives en contribuant à évaluer la situation et à sensibiliser les acteurs politiques locaux et internationaux. Une des premières évaluations fut initiée en Ouganda en 1989 par une antenne britannique de l'association internationale Save the children, en partenariat avec le ministère du Travail et des Affaires sociales. L'étude menée par Hunter, qui fit l'objet du premier article publié sur la question des « orphelins du sida» en Afrique subsaharienne [1990], mit en évidence le nombre élevé d'orphelins dans certaines régions ougandaises.

En quelques années, d'une simple dénomination servant à caractériser une réalité socioépidémique, les « orphelins du sida » sont devenus une catégorie institutionnelle qui allait structurer et conditionner les politiques nationales et internationales de lutte contre le sida ciblant les enfants. Tandis que les associations locales s'employaient à répondre aux problèmes de terrain en apportant des aides financières, alimentaires et psychosociales aux enfants orphelins, les agences onusiennes se sont engagées dans la réponse apportée à la «crise des orphelins » à travers l'organisation de rencontres et de groupes de travail favorisant le partage d'expériences et le renforcement des capacités. Une des premières rencontres associant des représentants internationaux et africains a été organisée en 1991 à Florence sous l'égide de l'Unicef. Dans le domaine de la coopération bilatérale, certains pays occidentaux comme les États-Unis ont également répondu à la « crise des orphelins », généralement en lien avec des agences internationales. En 1992, le gouvernement américain a initié la plateforme Aids orphans task force, qui réunissait les autorités politiques américaines, des ONG nationales et internationales, le Displaced children and orphans fund et la Banque mondiale. L'année suivante, les premiers financements ciblant les enfants étaient débloqués en faveur principalement des pays anglophones d'Afrique centrale et australe. En matière de politiques nationales, au cours des années 1990, de rares pays, essentiellement à forte prévalence comme l'Ouganda ou le Malawi, ont développé des politiques proactives répondant à la «crise des orphelins du sida ». Au Malawi, le gouvernement a organisé en 1991 une consultation nationale qui a débouché sur la création de la National orphan care task force, la rédaction d'un guide national de prise en charge en 1992, puis la création d'un programme national en 1996. À partir de 1997, des estimations nationales du nombre d'enfants orphelins du sida ont été produites sous l'égide de l'Usaid, de l'Unicef et de l'Onusida. Amplement médiatisés, quatre rapports publiés entre 1997 et 2004 [Hunter, Williamson, 1997 ; 2000 ; Unaids, Unicef, Usaid, 2002 ; 2004] deviennent des références institutionnelles majeures, offrant les bases du cadre stratégique qui va piloter la mise en place des politiques internationales et nationales de lutte contre le sida ciblant les enfants [Unicef, 2004]. 


\section{Controverses et critiques de la catégorie des «orphelins du sida »}

À partir de la fin des années 1990, la focalisation des politiques et des programmes d'intervention sur les « orphelins du sida » a suscité de vives critiques. Premièrement, c'est la définition même de la notion d'orphelin qui a prêté à controverses. Suivant les variables retenues, notamment l'âge des enfants et la prise en compte du décès d'un ou des deux parents -, les définitions de ce qu'était un orphelin variaient en effet d'un pays à un autre, et d'une institution à une autre, rendant les comparaisons longitudinales et géographiques difficiles. Les premiers chiffres produits sous-estimaient l'ampleur du phénomène, car ils n'intégraient pas les orphelins de père et les adolescents âgés de 15 à 18 ans [Unaids, Unicef, Usaid, 2002]. Deuxièmement, les critiques ont porté sur l'effet potentiellement stigmatisant de politiques et de programmes ciblant principalement les enfants de parents décédés des suites du sida. Mis en œuvre au niveau des communautés caractérisées par un niveau élevé d'interconnaissance et une forte visibilité sociale de l'épidémie, le ciblage des orphelins pouvait susciter des discriminations, voire le rejet de l'enfant de la famille, de l'école et des espaces sociaux [Deacon, Stephney, 2007]. Troisièmement, c'est le caractère électif des programmes ciblant principalement les « orphelins du sida » qui a été remis en question. Ceux-ci ont incarné une catégorie-écran éludant la réalité et la diversité des situations vécues par les enfants dont les parents vivent avec le VIH autant que les enfants euxmêmes infectés par le VIH [Meintjes, Giese, 2006 ; Unaids, Unicef, Usaid, 2004]. Cette fragmentation de la réponse, amplifiée par la multiplication des acteurs et des programmes mis en ouvre sans concertation systématique, a alimenté une concurrence des souffrances entre les enfants subissant l'impact de l'épidémie, dont certaines dimensions chez les enfants ont été prises en compte tardivement. Les enfants infectés par le VIH sont ainsi longtemps restés la «tache aveugle » de l'épidémie [Elenga et al., 2006; Wong et al., 2008]. De même, les enfants affectés par le VIH ont été peu ciblés durant les premiers temps de l'épidémie, alors qu'ils pouvaient également être confrontés aux mêmes problèmes de stigmatisation, de malnutrition ou de déscolarisation que les orphelins.

\section{Émergence et institutionnalisation de la catégorie des OEV}

En réponse aux critiques adressées à la catégorie des orphelins du sida, les agences internationales ont redéfini la notion d'enfant orphelin en intégrant, à partir de 2002, les orphelins de père, puis en modifiant en 2004 les âges de référence afin d'intégrer les adolescents âgés de 15 à 18 ans [Unaids, Unicef, Usaid, 2004, p. 7]. De nouvelles dénominations restituant le caractère multidimensionnel de l'impact de l'épidémie sur les enfants et limitant la stigmatisation ont également été instituées. Les orphelins du sida ont ainsi été renommés les « orphelins à cause du VIH/sida », ou les enfants rendus orphelins par le VIH/sida. C'est la catégorie des OEV qui s'est imposée et a été institutionnalisée à partir des années 2000. S'il est difficile de dater précisément l'apparition de cette catégorie, la dénomination orphans and vulnerable children figure dans plusieurs rapports et évaluations produits dans des pays comme la Zambie ou l'Ouganda, pays 
particulièrement touchés par l'épidémie à la fin des années 1990. En 2000, l'expression orphelins et enfants affectés par le sida est également utilisée dans le rapport de référence édité par l'Unicef et Usaid [2000]. La catégorie des OEV sera institutionnalisée au niveau international à partir de 2001 à travers la Déclaration d'engagement sur le VIH/sida adoptée en juin 2001, lors de la session extraordinaire de l'Assemblée générale de l'ONU évoquée en introduction.

La catégorie des OEV a remplacé celle des « orphelins du sida » avec l'objectif plus large de répondre aux besoins des « orphelins et autres groupes d'enfants qui sont plus exposés aux risques que leurs pairs », pour reprendre la définition de la Banque mondiale [2006]. En pratique toutefois, l'opérationnalisation de la catégorie des OEV a principalement ciblé des enfants en lien avec l'épidémie du sida du fait, notamment, de la mobilisation politique internationale qui caractérise l'exceptionnalisme de l'épidémie et de l'ampleur des financements collectés avec la création du Fonds mondial pour le VIH/sida, la tuberculose et le paludisme en 2002 et du President's emergency plan for aids relief en 2003. La catégorie des $\mathrm{OEV}$ a ainsi donné lieu à une transnationalisation de l'action publique qui s'est traduite par l'adoption de politiques nationales destinées aux OEV dans plus de vingt-cinq pays subsahariens. Des indicateurs comme l'index OPPEI (OVC policy and planning effort index) préconisé par l'Onusida, qui vise à évaluer la progression de la réponse nationale concernant les $\mathrm{OEV}$, ont également été instaurés.

À la fin des années 2000 , la catégorie des OEV va à son tour faire l'objet de critiques. Les millions d'enfants concernés associés à la diversité des situations socioéconomiques, des contextes politiques et des trajectoires individuelles rendent difficilement opérationnelle la notion de vulnérabilité [Skinner et al., 2006]. Dans les contextes de pauvreté généralisée qui caractérisent les pays subsahariens, la question de la «concurrence des souffrances »n'existe pas seulement entre les enfants subissant directement l'impact de l'épidémie, mais joue plus généralement au sein des communautés socioéconomiques où évoluent ces enfants. L'accès aux soins et à l'éducation ou la malnutrition sont autant de problèmes socioéconomiques et de santé auxquels sont confrontés la majorité des enfants d'une communauté [Sherr et al., 2008]. Ainsi, si l'existence d'une vulnérabilité spécifique des enfants liée à l'infection à VIH est reconnue, notamment en matière de stigmatisation et d'accès aux soins, les réponses conjoncturelles relevant de l'urgence et de l'aide humanitaire mises en place dans les premiers temps montrent leurs limites. Par ailleurs, les programmes développés autour de la catégorie des OEV ont pu avoir des effets délétères en créant un système de captation des ressources par des personnes autant que des associations [Delpeu, 2011 ; Guillermet, 2008].

En réponse aux travaux menés par l'Inter-agency task teams on children and HIV/aids de l'Unicef et la Joint learning initiative on children and aids (JLICA) ${ }^{8}$,

8. Créé en 2006, le projet JLICA est une alliance indépendante et à durée limitée de chercheurs, praticiens, activistes, et décideurs qui a réalisé une recherche opérationnelle de large envergure ayant mis en évidence la nécessité de placer les enfants et les familles au centre des programmes, au lieu de simplement cibler les « enfants vulnérables et les orphelins » [Irwin, Adams, Winter, 2009]. 
deux évolutions importantes ont marqué les politiques de lutte contre le sida ciblant les enfants à la fin des années 2000. Premièrement, un consensus prescrivant que la réponse apportée à l'impact de l'épidémie sur les enfants devait être sensible au VIH/sida et non pas exclusive a émergé, rompant avec les approches segmentaires relevant de l'urgence et de l'aide humanitaire. Deuxièmement, la nécessité de développer une réponse plus structurelle à travers l'instauration de systèmes de protection sociale sensibles aux enfants, incluant ceux qui subissent l'impact de l'épidémie a été retenue [Yates, Chandan, Lim, 2010]. Finalement, ces évolutions marquent l'intégration de la nécessité de dépasser la réponse conjoncturelle relevant de l'aide d'urgence portée par les associations qui s'est focalisée sur les orphelins du Sida, au profit d'une approche structurelle mise en œuvre par l'État pour développer la protection sociale des enfants.

\section{La concurrence des souffrances : une famille à l'épreuve de la catégorie des OEV}

À partir d'une étude de cas portant sur une famille affectée par le VIH, cette partie décrit empiriquement le caractère électif et segmentaire des programmes ciblant les enfants qui alimentent la «concurrence des souffrances». Sont ici décryptés les logiques et les processus institutionnels suivant lesquels une mère et les membres d'une fratrie accèdent aux soins et aux prestations sociales ou $a$ contrario en sont exclus, suivant des critères biologiques d'âge et de statut virologique, en l'occurrence le fait d'être infecté par le VIH.

Âgée de 39 ans, Victoire est mère de quatre enfants dont deux sont infectés par le VIH. Elle découvre sa séropositivité en novembre 2004 à Bobo-Dioulasso, à la suite d'un test de dépistage effectué lors d'une visite prénatale à six mois de grossesse. Née en Côte d'Ivoire de parents burkinabè d'origine Lobi, Victoire avait fui quatre mois auparavant son pays natal à la suite de la crise politique, à l'instar de milliers d'autres diaspos ${ }^{9}$. Son mari étant resté en Côte d'Ivoire, elle se retrouve à élever seule et sans ressources financières durables ses trois filles, dans un pays qu'elle découvre et où les réseaux sociaux et familiaux qu'elle pouvait mobiliser sont limités en raison de la stigmatisation et du secret associés au sida [Hejoaka, 2009].

Trois mois après la découverte de son statut, Victoire accouche d'un garçon dont elle redoute pendant 18 mois qu'il soit infecté par le VIH. En effet, si en 2005 au Burkina Faso la prévention de la transmission du VIH de la mère à

9. Diaspo est une expression dérivée de diaspora couramment utilisée en Côte d'Ivoire et au Burkina Faso pour désigner les migrants burkinabè de seconde génération nés en Côte d'Ivoire. Considérée comme un réservoir de main-d'œuvre par l'Administration coloniale française, la population burkinabè a alimenté en force de travail les colonies françaises de l'Afrique occidentale. Dans les années post-indépendance, la dynamique migratoire s'est accentuée, s'inscrivant dans le contexte d'une forte croissance économique qui a favorisé le regroupement familial. Mais la crise économique des années 1970, qui a particulièrement touché l'économie de plantation ivoirienne, s'est traduite par une paupérisation des populations, engendrant un retour massif de Burkinabè et une recomposition des rapports sociaux [Zongo, 2003]. 
l'enfant (PTME) était théoriquement accessible dans $67 \%$ des districts, d'un point de vue opérationnel, peu d'enfants exposés bénéficiaient effectivement d'un continuum de prise en charge allant du traitement prophylactique, à la confirmation du statut sérologique à 18 mois, voire à l'initiation précoce d'un traitement antirétroviral lorsque les enfants étaient infectés. En 2005, conformément aux recommandations de l'OMS, les protocoles nationaux de PTME prescrivaient l'administration d'une dose de Nevirapine ${ }^{\odot}$ au moment de l'accouchement, tandis qu'en Europe, la prophylaxie recommandée consistait en un traitement préventif par traitement antirétroviral durant la grossesse. Ce type de prophylaxies était alors en train d'être évalué dans le cadre d'un essai clinique à Bobo-Dioulasso ${ }^{10}$. Mais enceinte de plus de trois mois, Victoire ne put intégrer l'essai faute de remplir les critères d'inclusion. En l'absence d'une politique systématique de dépistage précoce des enfants nés de mères vivant avec le VIH, Victoire dut attendre que son fils, alors catégorisé comme enfant exposé au VIH, ait dix-huit mois pour apprendre qu'il n'était pas infecté ${ }^{11}$. Ce défaut de prise en charge des nourrissons et des jeunes enfants reflète l'épidémie marginale qu'a été l'infection à VIH pédiatrique jusqu'au milieu des années 2000 , les politiques publiques internationales ciblant principalement les orphelins du sida.

Victoire fit dépister ses trois filles: Sophie âgée de 6 ans, Florence âgée de 12 ans et Jeanne âgée de 15 ans. La benjamine se révéla être séropositive au VIH comme l'aînée, infectée pour sa part par voie sexuelle. Au regard des catégories institutionnelles, Sophie et Jeanne étaient considérées comme des enfants infectées, tandis que Florence relevait de la catégorie des enfants affectés. En pratique, au niveau des associations communautaires et acteurs de la lutte contre le sida, les enfants étaient communément désignés par le terme générique d'OEV qui faisait abstraction des spécificités médicales ou familiales les caractérisant. La prise en charge des OEV était devenue en 2005 au Burkina Faso une composante programmatique du plan stratégique 2006-2010 de lutte contre le VIH/sida et les IST. Si des programmes et des prestations ont été financés et mis œuvre, ceux-ci, souvent médiatisés à travers la distribution de kits scolaires ou de vivres aux OEV, ont toutefois touché un nombre limité d'enfants. En 2010, le rapport de l'UNICEF sur l'état d'avancement des politiques publiques ciblant les enfants face au sida indiquait ainsi qu'au Burkina Faso, moins de $7 \%$ des familles dans lesquelles résidaient des $\mathrm{OEV}$ avaient bénéficié d'un soutien matériel ou psychologique [Unicef, 2010].

10. La ville de Bobo-Dioulasso est le site du centre de recherche scientifique Muraz, fondé en 1939. Centre d'excellence dans la sous-région ouest-africaine, il est partenaire de nombreuses recherches scientifiques médicales.

11. Chez les adultes et les enfants âgés de plus de 18 mois, le dépistage du VIH implique de détecter des anticorps dirigés contre les divers constituants du VIH à travers l'utilisation de tests ayant un faible coût. Chez les enfants âgés de moins de 18 mois, ceux-ci étant porteurs des anticorps de leur mère, un examen biologique (Polymerase chain reaction ou PCR) est nécessaire. Généralisée dans les pays du Nord depuis la fin des années 1990, l'utilisation de la PCR dans le cadre du diagnostic précoce d'enfants nés de mères vivant avec le VIH n'est pas systématiquement accessible dans les pays subsahariens. 
Au niveau de la population, la catégorie institutionnelle des OEV était peu connue dans les années 2000 contrairement à celle des "orphelins du sida», devenue une catégorie populaire sous l'effet croisé de leur médiatisation et de la visibilité sociale de l'épidémie de sida à Bobo-Dioulasso. Pour ce qui concerne les enfants infectés et affectés, ceux-ci ne s'identifiaient pas aux catégories institutionnelles précédemment évoquées, ces expressions n'étant pas utilisées pour s'adresser à eux. Par contre, les enfants vivant avec le VIH, informés de leur statut ou orphelins étaient généralement conscients des «identités blessées » pour reprendre l'expression de Pollak auxquelles renvoyaient leur maladie ou le décès de leurs parents [1993]. Au cours des entretiens réalisés avec les enfants, la notion de vulnérabilité paraît méconnue et donc, pas signifiante. Par contre, les enfants se référaient régulièrement, avec leurs propres mots, au contexte de pauvreté dans lequel ils vivaient, évoquant le manque d'argent, la difficulté des parents à payer les frais de scolarité, la rareté et la non-diversité de l'alimentation ou encore le défaut de moyens de transport, nombre d'enfants devant parcourir les kilomètres entre leur domicile, l'hôpital et l'association à pied. Régulièrement exposés aux discours sociaux et médiatiques moralisateurs et mortifères produits sur le VIH/ sida, les enfants évoquaient également la stigmatisation à laquelle ils étaient régulièrement confrontés. Certains d'entre eux étaient aussi victimes d'insultes et de discriminations directes de la part d'adultes ou d'autres enfants [Hejoaka, 2012].

Les catégories institutionnelles, en opérant un triage entre les enfants ayant accès à des programmes et ceux qui en sont exclus, ont des conséquences directes sur leur vie. Les prestations de base dispensées (soins, scolarité, alimentation) relèvent en effet de l'exception et du privilège dans un pays où la moitié de la population vit en dessous du seuil de pauvreté. Ainsi, Sophie, la benjamine des trois sœurs fut mise sous traitement antirétroviral à l'âge de 7 ans et demi dans le cadre d'un essai clinique mené dans le service de pédiatrie où elle était suivie. Au Burkina Faso où les premiers patients ont été mis sous traitement à partir de 1999, achetant à titre personnel des ARV auprès de la Centrale d'achat des médicaments essentiels génériques et des consommables, le ministère de la Santé a institué en 2002 l'Initiative burkinabè d'accès aux ARV, qui a permis de développer la dispensation d'ARV (pour adultes et enfants) dans le cadre de programmes pilotes. À partir de 2003, les ARV ont commencé à être distribués à l'échelle nationale dans des centres de référence et quelques associations de prise en charge spécialement accréditées. Les ARV pédiatriques ont été dès lors gratuits, contrairement à ceux dispensés aux adultes, qui ne l'ont été qu'en 2010. Toutefois, l'accès des enfants aux ARV est resté limité, révélant de persistantes inégalités générationnelles. Ainsi, en 2013, en dépit des nombreux progrès réalisés, seulement $10 \%$ des enfants burkinabè contre $43 \%$ des adultes ayant besoin d'un traitement antirétroviral y avaient accès [Unicef, 2013].

Bien que les ARV aient été gratuits, la participation à cet essai permettait à Sophie de bénéficier gratuitement d'examens biologiques et de médicaments traitant les infections opportunistes, d'être accompagnée individuellement au niveau psychosocial par des conseillères communautaires et de percevoir pour 
le transport à l'hôpital une compensation financière (régulièrement réallouée par leur mère à l'alimentation). Dans le contexte du Burkina Faso, pays où les médicaments et les actes de soins demeurent à la charge des patients et des familles, les soins et les traitements auxquels Sophie avait accès en tant qu'enfant infectée lui octroyaient paradoxalement un statut sanitaire privilégié, comparativement aux autres membres de sa fratrie. Ainsi, atteinte d'un accès palustre, sa sœur cadette ne fut admise à l'hôpital qu'après trois jours de forte fièvre, sa mère n'ayant pas les moyens de payer les frais d'hospitalisation et ne maitrisant pas les démarches à entreprendre pour bénéficier de l'exemption des frais d'hospitalisation réservée aux personnes considérées comme indigentes. Quant à l'otite chronique que présenta son petit frère à l'âge de 3 ans ${ }^{12}$, elle fut traitée par recours à la médecine traditionnelle, application de cataplasmes de plantes sur l'oreille, à défaut de pouvoir payer une visite médicale et les médicaments prescrits dans le système de santé moderne. Pour sa part, Victoire ne fut mise sous traitement antirétroviral qu'en 2012, à un stade avancé de la maladie, l'accès aux ARV étant limité par la lourdeur des processus et le nombre de patients en attente d'un traitement. Grabataire durant plusieurs mois, Victoire frôla la mort et dut arrêter le travail de femme de ménage qu'elle exerçait occasionnellement et qui procurait une source de revenus complémentaires à la famille. Ce sont ces filles qui s'occupèrent alors d'elle, effectuant un travail invisible de care rarement pris en compte dans les politiques internationales de lutte contre le sida [Evans, Becker, 2009].

$\mathrm{Au}$ sein de l'association communautaire dont sa mère était membre, Sophie bénéficia également, en tant qu'enfant infectée, d'un parrainage thérapeutique alloué par une association caritative française finançant notamment ses activités à travers des collectes de fonds. Cette somme mensuelle de 15000 francs CFA ${ }^{13}$ bénéficiait à l'ensemble de la famille, dont elle était la seule ressource financière régulière, et était principalement destinée à l'achat de nourriture. En effet, les dotations alimentaires (essentiellement du riz, de la farine de maïs et de l'huile) dont Victoire et ses filles bénéficiaient du fait de leur statut sérologique ne couvraient qu'une partie des besoins de la famille. Ces dotations, visant à l'origine à améliorer l'état nutritionnel des adultes et enfants infectés par le VIH, profitaient à l'ensemble de la famille, voire dans certains cas à des proches ou des voisins. Enfin, les trois sœurs, toujours dans le cadre des prestations psychosociales dispensées par l'association communautaire, bénéficièrent durant plusieurs années d'aides scolaires à travers un programme national d'appui à la scolarité ciblant les OEV. Après l'instauration de la gratuité de la scolarité dans les établissements publics au Burkina Faso en septembre 2007, de nombreux partenaires internationaux suspendirent les financements alloués à la scolarité, ce qui mit fin aux aides de Florence (qui n'est pas infectée), puis les années suivantes des deux autres

12. Si les enfants ont fait l'objet d'un traitement marginal dans les politiques de lutte contre le sida, les jeunes enfants [2-8 ans] ont été particulièrement occultés [Dunn, 2005].

13. $22,87 €$. 
sœurs, scolarisées dans des établissements privés au coût plus élevé, faute de place dans l'enseignement public.

L'autre point révélé par l'étude de cette fratrie est l'approche monolithique qui est communément faite des enfants, les spécificités des âges ou du genre n'étant pas prises en compte dans la conception des programmes. Sous le vocable d'enfant, ce sont non seulement les jeunes enfants, mais également les adolescents voire les jeunes adultes qui sont désignés, leur point commun étant d'être des cadets sociaux, c'est-à-dire des non-adultes. Les données épidémiologiques en revanche définissent d'un point de vue statistique les enfants comme étant âgés de 0 à 14 ans. Les adolescents âgés de plus de 15 ans sont intégrés à la catégorie des adultes, ce qui alimente leur invisibilité politique et programmatique. Dans le cas de la famille de Victoire, Jeanne, l'aînée de la fratrie tomba enceinte en 2006 alors qu'elle venait d'avoir seize ans. Considérés comme des enfants, les adolescents n'avaient pas alors accès à des programmes de santé sexuelle et reproductive. Le «fiancé » de Jeanne, un jeune âgé de 19 ans, ne voulant pas reconnaître la grossesse, Jeanne passa brusquement du statut d'enfant à celui de mère célibataire, bien qu'elle demeurât financièrement à la charge de sa mère. Elle fut dès lors suivie dans le programme des adultes et bénéficia d'une aide financière exceptionnelle sur un fonds spécial de l'association communautaire, aucun programme existant ne ciblant spécifiquement les adolescents qui vivent avec le VIH. Il faudra attendre le début de la décennie 2010 pour que la question des adolescents et des jeunes adultes vivant avec le VIH soit inscrite sur l'agenda des politiques internationales [Who, 2014]. Finalement, l'histoire de Jeanne montre l'importance de penser l'aide internationale en matière de recherche autant que d'interventions opérationnelles à l'intersection de l'âge et du genre, ces deux composantes à la fois biologiques, sociohistoriques et culturelles se façonnant réciproquement.

\section{Trois leçons sur les enfants dans les programmes de développement à partir du cas de l'épidémie du $\mathrm{VIH} /$ sida}

La question des enfants dans les programmes d'aide internationale dédiés à la santé et à l'enfance constitue un objet d'étude multidimensionnel et complexe qui ne peut être synthétisé dans le cadre d'un article. Toutefois, trois leçons peuvent être tirées de la fabrique et des usages politiques et moraux des catégories institutionnelles ciblant les enfants dans le contexte de l'épidémie du sida.

\section{Pour une approche générationnelle des politiques de développement}

Alors que l'aide internationale a longtemps été appréhendée sur le plan des inégalités socioéconomiques internationales, puis plus récemment de genre, elle a été peu pensée au prisme de l'âge alors même que les enfants sont une composante démographique majeure dans les pays des Suds. Étudier les enfants dans le contexte de l'aide internationale implique d'adopter une approche générationnelle, à la suite des travaux initiés par Mayall [2002]. S'inspirant du processus de 
« genrisation» des gender studies, la sociologue britannique propose le concept de generationing processes, que je propose de traduire par le néologisme «processus de générationalisation ». Il s'agit de s'intéresser aux processus relationnels suivant lesquels les personnes sont reconnues comme des enfants et à travers lesquels les enfants et l'enfance acquièrent certaines caractéristiques liées au contexte local et aux transformations sociales. Développer une approche générationnelle nécessite ainsi d'entrecroiser trois niveaux d'analyse. Il s'agit d'abord de recentrer l'analyse sur les enfants et les adolescents considérés en tant que membres d'un groupe social spécifique structuré par l'âge. Cela implique d'articuler les approches constructivistes, décryptant les discours et les constructions politiques et morales sur l'enfance dans un contexte de globalisation à des analyses microsociales s'intéressant à l'enfant en tant qu'acteur social. Il est important ensuite de s'intéresser aux enfants en tant que génération spécifique, c'est-à-dire un ensemble d'individus nés au cours d'une même période et dont la socialisation a été marquée par les mêmes événements. Peut ici être analysé le traitement singulier des différents âges de la vie en décryptant les inégalités générationnelles qui sous-tendent l'aide internationale. Les privilèges que la condition d'enfant représente en matière d'accès à des programmes de plus en plus focalisés sur les enfants, ou a contrario les barrières rencontrées dans le cadre de programmes adulto-centrés qui occultent la singularité des besoins des enfants et de la réponse à apporter, peuvent ici être explorés. L'épidémie du sida, longtemps caractérisée par son exceptionnalisme, offre un exemple emblématique à ce sujet. Les enfants ont de facto eu accès à des programmes structurés autour des catégories décrites préalablement, des prestations économiques et psychosociales ou des traitements antirétroviraux coûteux nécessitant un plateau technique de qualité. Mais comparativement aux adultes, ces réponses ont été mises en place avec un décalage d'une dizaine d'années. Il existe par ailleurs d'importantes inégalités en matière d'accès au dépistage, aux traitements antirétroviraux ou à l'information ${ }^{14}$. Enfin, il est important d'adopter une approche relationnelle des générations en s'intéressant aux rapports sociaux d'âge entre les enfants et les adultes. Ici, les problématiques telles que les approches familiales mises en place dans les programmes d'aide internationale, les relations des professionnels, formés ou non aux métiers de l'enfance, aux enfants ou encore la façon dont les enfants s'approprient de nouveaux dispositifs inspirés de ce qui se fait chez les adultes peuvent être explorées.

\section{La concurrence des souffrances : triage et programmes de développement}

Les programmes ciblant les enfants mis en œuvre dans la réponse internationale apportée à l'épidémie de sida répondent à de multiples besoins, accès aux soins et aux traitements, alimentation, scolarisation, aide psychosociale, etc., et viennent pallier en partie les politiques et les programmes étatiques structurellement faibles

14. En 2013, en Afrique subsaharienne, $24 \%$ des enfants ayant besoin d'un traitement antirétroviral $\mathrm{y}$ avaient accès, contre $47 \%$ des adultes [Unaids, 2014]. 
en matière de santé, d'éducation et de protection sociale. Or, comme cela a été décrit dans la littérature [Atlani-Duault, Dozon, 2011], l'aide internationale s'est transformée ces deux dernières décennies, avec non seulement un accroissement des actions humanitaires, mais également la multiplication des intervenants et des bailleurs, ONG locales et internationales, agences onusiennes, associations de solidarités internationales, plan présidentiel, fondations, etc. L'un des pendants de l'aide internationale est le morcellement des interventions, qui participe à la «concurrence des souffrances » décrite dans cet article. Au-delà de l'étude des constructions modernes de l'enfance et des enfants comme bénéficiaires, les anthropologues devraient également s'intéresser à ceux qui sont exclus des dispositifs d'aide. Appréhender, sous un angle large, l'aide internationale ciblant les enfants constitue des enclaves sociosanitaires apportant des réponses conjoncturelles et contingentes à des problèmes d'ordre structurel, notamment liés au sousdéveloppement économique et à la faiblesse des institutions étatiques. Saisis dans une perspective morale et politique, les travaux de Nguyen [2010] sur la «médecine du tri », qui décrivent à partir du concept de citoyenneté thérapeutique les processus biopolitiques suivant lesquels les malades ont accès ou non aux soins et aux traitements, offrent un cadre conceptuel heuristique pour penser la construction des catégories institutionnelles et leurs effets sur la vie des enfants. Appliqué à d'autres domaines que la médecine, le triage, en l'occurrence institutionnel, qui s'opère à travers les catégories interventionnelles, permet de penser les programmes de développement en décrivant ceux qui en bénéficient autant que ceux qui en sont exclus. Comme le suggère la « concurrence des souffrances » ici décrite au sein d'une même famille ou d'une communauté, la frontière entre les bénéficiaires et les exclus n'est pas réductible à une frontière binaire déterminée par les classes sociales ou les niveaux de vulnérabilité départageant les pauvres et les riches, mais dépend de la construction politique et sociale des catégories sur des critères biologiques et familiaux. En produisant des cadres de classification et d'action sur le monde social, les catégories institutionnelles constituent des enjeux stratégiques de pouvoir dans le contexte de pauvreté généralisée qui caractérise les pays subsahariens.

\section{Dépasser une vision monolithique de l'enfant dans les programmes de développement}

La problématique de l'enfant dans les politiques d'aide internationale est souvent traitée de façon monolithique et naturaliste, faute de travailler à l'intersection entre les âges de l'enfance, le genre et les catégories sociales. La classification des enfants dans la catégorie des OEV s'est traduite par la focalisation sur la dimension sociale de l'épidémie, négligeant les enfants infectés [Elenga et al., 2006 ; Hejoaka, 2012]. Les catégories internationales peinent à prendre en compte la singularité des situations épidémiologiques et socioéconomiques de chaque pays. La prise en charge des enfants orphelins pose des problèmes différents dans des pays à forte prévalence comme l'Afrique du Sud ou le Zimbabwe, par comparaison avec des 
pays comme le Sénégal, où l'épidémie n'est pas généralisée ${ }^{15}$. La question des enfants dans les programmes d'aide devrait également mieux tenir compte de la spécificité des âges de la petite enfance, de l'enfance, de l'adolescence, voire des jeunes adultes. Le cas des adolescents vivant avec le VIH illustre ce défaut de considération des âges de l'enfance. Les politiques de lutte contre le sida ont principalement ciblé les adolescents à travers les programmes de prévention, négligeant la prise en charge et l'information des adolescents. Ainsi en 2013, le VIH/sida était la première cause de mortalité chez les adolescents en Afrique subsaharienne [Who, 2014]. Par ailleurs, les programmes intégrant la santé sexuelle et reproductive pour les adolescents vivant avec le VIH sont peu développés à une échelle nationale. Les programmes étant structurés autour des catégories d'âge, il convient également de mieux analyser ce qui se joue aux frontières de ces âges, en documentant le passage d'un âge à un autre. Or, la majorité légale, à l'âge de 18 ans conformément à la Convention internationale des droits de l'enfant de 1989, constitue souvent un critère d'exclusion ou de sortie de programme, impliquant la fin des prestations ou de l'accès gratuit aux soins. Enfin, la diversité des enfants nécessite d'être abordée en termes de genre et en faisant porter les analyses autant sur les enfants que sur les relations générationnelles. L'éducation et les soins des enfants sont autant d'activités domestiques relevant du care communément dévolues aux femmes [Hejoaka, 2011]. Penser les enfants dans les programmes de développement invite donc à adopter une approche relationnelle à l'intersection du genre, de l'enfance et du développement.

\section{Bibliographie}

Atlani-Duault L., Dozon J.-P. [2011], « Colonisation, développement, aide humanitaire. Pour une anthropologie de l'aide internationale », Ethnologie française, vol. 41, n 3, p. 393-403.

Atlani-Duault L., Vidal L. [2009], Anthropologie de l'aide humanitaire et du développement: des pratiques aux savoirs, des savoirs aux pratiques, Paris, Armand Colin, $230 \mathrm{p}$.

BONNET D. [2012], « The absence of the child in ethnology : a non-existent problem ? ", AnthropoChildren, vol. 1, numéro spécial «Anthropologie de l'enfance et des enfants à travers le monde », 8 p.: http://popups.ulg.ac.be/AnthropoChildren/docannexe.php?id=931\%5D (page consultée le 20 juin 2014).

DeAcon H., StePhney I. [2007], HIV/aids, stigma and children : A literature review, HSRC press Cape Town, South Africa, 87 p.: http://www.hsrcpress.ac.za/product.php? productid=2197 (page consultée le 2 juillet 2014).

DelPeu M. [2011], «Prendre en charge les enfants affectés par le VIH/sida en Inde du Sud. Recommandations internationales et pratiques locales », in Евоко F., BRoQUA C., BOURDIER F. (dir.), Les Suds face au Sida: quand la société se mobilise, Marseille, IRD. p. $179-203$.

15. Une épidémie est dite généralisée lorsque la prévalence du VIH (soit la proportion de personnes infectées par le VIH) dépasse $1 \%$ dans la population générale adulte (15-49 ans). 
DunN A. [2005], «HIV/aids : what about very young children?», Working papers in early childhood development, The Hague, The Netherlands, Bernard van Leer foundation, $72 \mathrm{p}$.

Elenga N., Fassinou P., Dossou R., Aka Dago-Akribi H., Msellati P. [2006], « La tache aveugle de l'infection par le VIH en Afrique : la prise en charge de l'infection pédiatrique. L'expérience d'un programme pilote, le programme enfant yopougon », in ADJAMAGBO A., Msellati P., Vimard P. (dir.), Santé de la reproduction et fécondité dans les pays du Sud. Nouveaux contextes et nouveaux comportements, Bruxelles, Academia, p. 332-359.

Evans R., BeCKeR S. [2009], Children caring for parents with HIV and Aids : global issues and policy responses, Bristol U.K., Policy press, $350 \mathrm{p}$.

Foster G., Germann S. [2002], «The orphan crisis », in Essex M., Mboup S., Kanki P.J., Marlink R.G., Tlou S.D., Holme M. (eds), Aids in Africa, New York, Springer, p. 664-675.

GuilLERMET E. [2008], «Les constructions de l'orphelin. Une anthropologie de l'enfance globalisée entre Islam, malnutrition et sida, à Zinder (Niger) », Thèse de doctorat en anthropologie sociale, université de Lyon II, 214 p.

HEJOAKA F. [2009], « Care and secrecy : being a mother of children living with HIV in Burkina Faso », Social science \& medicine, vol. 69, n 6, p. 869-876.

HeJoAKa F. [2011], «La solitude des femmes dans le traitement et l'accompagnement des enfants vivant avec le VIH au Burkina Faso », in Desclaux A., Msellati P., Sow K. (dir.), Les Femmes à l'épreuve du VIH dans les pays du Sud. Genre et accès universel à la prise en charge, Paris, ANRS, p. 193-205.

HEJOAKA F. [2012], «L'enfant gardien du secret. Vivre et grandir avec le sida et ses traitements à Bobo-Dioulasso (Burkina Faso) », Thèse d'anthrolopogie sociale et ethnologie, EHESS, 423 p. : http://tel.archives-ouvertes.fr/tel-00761339/ (page consultée le 15 juin 2014).

HuNTER S.S. [1990], «Orphans as a window on the Aids epidemic in sub-saharan Africa: initial results and implications of a study in Uganda », Social science \& medicine, vol. 31, $\mathrm{n}^{\circ}$ 6, p. 681-690.

Hunter S.S., Williamson J. [1997], Children on the brink: strategies to support children isolated by HIV/Aids, Washington D.C., USaid, $56 \mathrm{p}$.

Hunter S.S., Williamson J. [2000], Children on the brink 2000. Executive summary, updated estimates and recommendations for intervention, Washington D.C., USaid, 27 p.

Irwin A., AdAms A., Winter A. [2009], « Home truths. facing the facts on children, Aids and poverty », Final report of the joint learning initiative on children and HIV/Aids, HarvardGeneva, JLICA, 83 p. : http://www.jlica.org/protected/pdf-feb09/Final JLICA Report-final. pdf (page consultée le 20 juin 2014).

JAMES A., PROUT A. [1990], Constructing and reconstructing childhood : contemporary issues in the sociological study of childhood, London, Falmer Press, 248 p.

Lallemand S., Le Moal G. [1981], «Un petit sujet », Journal de la société des africanistes, vol. $51, \mathrm{n}^{\circ} 1-2$, p. 5-21.

LANCY D. [2008], The Anthropology of childhood: cherubs, chattel, changelings, Cambridge, Cambridge university press, $466 \mathrm{p}$.

MAYAll B. [2002], Towards a sociology for childhood: thinking from children's lives, Philadelphia P.A., Open university press, $217 \mathrm{p}$.

MeINTJES H., GIESE S. [2006], « Spinning the epidemic : the making of mythologies of orphanhood in the context of Aids », Childhood, vol. 13, n ${ }^{\circ} 3$, p. 407-430.

NGUYEN V.K. [2010], The Republic of therapy : triage and sovereignty in west africa's time of Aids, Durham, Duke university press, $256 \mathrm{p}$. 
Nyambedha E.O., Wandibba S., Aagaard-Hansen J. [2003], « Changing patterns of orphan care due to the HIV epidemic in western Kenya », Social science \& medicine, vol. 57, $\mathrm{n}^{\circ} 2$, p. 301-311.

Onusida [2011], Guide de terminologie de l'Onusida, Genève, Onusida, 38 p.

PANDOLFI M. [2002], «"Moral entrepreneurs", souverainetés mouvantes et barbelés : le biopolitique dans les Balkans postcommunistes », Anthropologie et sociétés, vol. $26, \mathrm{n}^{\circ} 1$, p. 29-51.

Pandolfi M., CoRbet A. [2011], « De l'humanitaire imparfait », Ethnologie française, vol. 41, $\mathrm{n}^{\circ} 3$, p. $465-472$.

PollaK M. [1993], Une identité blessée : études de sociologie et d'histoire, Paris, Métailié, $416 \mathrm{p}$.

Sherr L., Varrall R., Mueller J., Richter L., Wakhweya A., Adato M., Belsey M., Chandan U., Drimie S., Haour-Knipe Victoria Hosegood M., Kimou J., Madhavan S., Mathambo V., Desmond C. [2008], « A systematic review on the meaning of the concept "Aids orphan" : confusion over definitions and implications for care », Aids care, vol. 20, $\mathrm{n}^{\mathrm{o}} 5$, p. $527-536$.

Skinner D., Tsheko N., Mtero-Munyati S., Segwabe M., Chibatamoto P., Mfecane S., Chandiwana B., Nkomo N., Tlou S., Chitiyo G. [2006], «Towards a definition of orphaned and vulnerable children », Aids and behavior, vol. 10, n 6, p. 619-626.

UnAIDS [2014], Gap report, Geneva, Unaids, 422 p. : http://www.unaids.org/sites/default/files/ media_asset/Unaids_Gap_report_en.pdf (page consultée le 17 août 2014).

UNAIDS, UNICEF, USAID [2004], Children on the brink 2004 : a joint report on new orphan estimates and a framework for action, Washington, USaid, 46 p. : http://www.unicef.org/ publications/cob_layout6-013.pdf (page consultée le 10 septembre 2014).

UNAIDS, UNICEF, USAID [2002], Children on the brink. a joint report on orphan estimates and program strategies, Washington, Usaid : http://www.usaid.gov/pubs/hiv_aids/childrenreport.pdf (page consultée le 10 septembre 2014).

UNICEF [2004], Cadre pour la protection, les soins et le soutien des orphelins et enfants vulnérables vivant dans un monde avec le VIH et le sida, New York, Unicef, 48 p.: http://www.unicef.org/aids/files/Framework_French.pdf (page consultée le 6 janvier 2011).

UNICEF [2010], Children with Aids : fifth stocktaking report, New York, Unicef, Onusida, OMS, FNUAP, 48 p. : http://www.unicef.org/french/publications/files/Children_and_Aids-Fifth_ Stocktaking_Report_2010_EN.pdf (page consultée le 15 décembre 2010).

UNICEF [2013], Towards an Aids-free generation, Children and Aids : sixth stocktaking report, New York, Unicef, $88 \mathrm{p}$.

UnICEF, UnAIDS [2000], Principles to guide programming for orphans and other children affected by HIV/Aids, New York, Unicef, $12 \mathrm{p}$.

Wells K. [2009], Childhood in global perspective, Cambridge, Polity press, 220 p.

Wно [2014], «Health for the world's adolescents : a second chance in the second decade», online report, s.p. : http://www.who.int/maternal_child_adolescent/documents/seconddecade/en (page consultée le 10 septembre 2014).

Wong V., Macleod I., Gilks C., Higgins D., Crowley S. [2008], « The lost children of universal access, issues in scaling-up HIV testing and counselling », Vulnerable children and youth studies, vol. $1, \mathrm{n}^{\circ} 1$, p. 44-55. 
World BANK [2006], «OVC core definitions », OVC toolkit, Washington D.C., The World Bank : http://info.worldbank.org/etools/docs/library/162495/howknow/definitions.htm (page consultée le 15 mai 2015).

YAtes R., Chandan U.K., Lim A.K.P. [2010], «Child-sensitive social protection. A new approach to programming for children affected by HIV and Aids », Vulnerable children and youth studies, vol. $5, \mathrm{n}^{\circ} 3$, p. 208-216.

Zongo M. [2003], « La diaspora burkinabè en Côte d'Ivoire », Politique africaine, vol. 90, n 2, p. 113-126. 